山्यRANÇAISE

$>\mathrm{DE}$

$\stackrel{1=1}{\simeq}$ PÉDAGOGIE
Revue française de pédagogie

Recherches en éducation

162 | janvier-mars 2008

Acquisitions et progressions scolaires : recherches en psychologie

\title{
FLORIN Agnès (dir.) \& VRIGNAUd Pierre (dir.). Réussir à L'école : les effets des dimensions conatives en éducation
}

Rennes : Presses universitaires de Rennes, 2007. - 223 p.

Jorge Valenzuela

(2) OpenEdition

Journals

Édition électronique

URL : http://journals.openedition.org/rfp/963

DOI : $10.4000 /$ rfp.963

ISSN : 2105-2913

Éditeur

ENS Éditions

Édition imprimée

Date de publication : 1 janvier 2008

Pagination : 144-145

ISBN : 978-2-7342-1118-1

ISSN : 0556-7807

Référence électronique

Jorge Valenzuela, «FLoRin Agnès (dir.) \& Vignaud Pierre (dir.). Réussir à L'école : les effets des

dimensions conatives en éducation ", Revue française de pédagogie [En ligne], 162 | janvier-mars 2008, mis en ligne le 29 septembre 2010, consulté le 22 septembre 2020. URL : http://

journals.openedition.org/rfp/963; DOI : https://doi.org/10.4000/rfp.963

Ce document a été généré automatiquement le 22 septembre 2020.

(C) tous droits réservés 


\section{FLORIN Agnès (dir.) \& VRIGNAUD Pierre (dir.). Réussir à L'école : les effets des dimensions conatives en éducation}

Rennes : Presses universitaires de Rennes, 2007. - 223 p.

Jorge Valenzuela

\section{RÉFÉRENCE}

FloRin Agnès (dir.) \& VRIGNAUd Pierre (dir.). Réussir à L'école : les effets des dimensions conatives en éducation. Rennes : Presses universitaires de Rennes, 2007. - 223 p.

1 Ensemble de communications présentées lors d'un colloque organisé par le laboratoire de psychologie, éducation, cognition, développement de l'université de Nantes en juin 2006, sous la responsabilité scientifique d'Agnès Florin et Pierre Vrignaud.

Les communications abordent divers sujets tels que la motivation, l'estime de soi, les compétences sociales, de même que la personnalité. Malgré leur apparente diversité, ces thématiques traitent d'un concept commun, celui de la conation (effort) visant la réussite scolaire. Cette notion a évolué en recouvrant plusieurs domaines et considère autre les aspects traditionnels que sont l'effort, la motivation, la planification et la décision, l'impact du social sur le conatif. Ces communications suggèrent d'envisager des stratégies visant à l'amélioration des apprentissages scolaires.

Même si le nombre élevé d'articles ainsi que la multiplicité des domaines compliquent la tâche pour les analyser séparément, on peut dire en général, que les contributions sont théoriquement et méthodologiquement claires, solides et bien documentées. Dans l'ensemble, elles montrent la variété des aspects que recouvre le phénomène complexe de l'apprentissage scolaire et la nécessité de considérer des approches complémentaires concernant la réussite scolaire. Dans ce cadre, ces articles indiquent 
des pistes intéressantes visant l'intervention au niveau scolaire. De plus, bien qu'il s'agisse d'articles scientifiques, ils fournissent au lecteur non spécialiste un panorama théorique suffisamment intelligible. Par contre, nous sommes surpris par la sous représentation de la variable motivation et des références aux travails récents dans ce domaine, vu son large développement dans le monde francophone et la centralité de la motivation comme variable conative.

4 Le contexte de cet ouvrage est présenté par A. Florin et P. Vrignaud, où à titre d'introduction, ils proposent une brève révision sur la notion de conation et des effets des dimensions conatives en éducation. Viennent ensuite les différentes contributions. Y. Forner, aborde en premier lieu un des sujets le plus classique du domaine du conatif : la motivation. Son travail La motivation à la réussite en éducation et en orientation, essaie de mettre en évidence, depuis la perspective d'Atkinson (1983), le besoin de considérer la motivation à la réussite.

6 Une deuxième variable typiquement conative, l'estime de soi, est travaillée par M. de Leonardis et $\mathrm{Y}$. Prêteur dans l'Expérience scolaire, estime de soi et valeur accordé à l'école à l'adolescence, où ils montrent le rôle des variables qui médiatiseraient la relation entre l'estime de soi et la performance scolaire.

7 Une troisième contribution, celle d'A. Florin et P. Guimard, Comportements scolaires en maternelle et parcours scolaires ultérieurs, présente le processus d'élaboration et de validation globale d'un questionnaire d'évaluation des compétences scolaires en moyenne section de maternelle et qui profite des connaissances privilégiées que les enseignants de maternelle ont de ces enfants, en montrant la valeur descriptive et prédictive du questionnaire et l'importance de considérer une telle mesure.

8 Par ailleurs, P. Guimard et al., dans leur travail, Surmonter les difficultés de lecture-écriture au collège, montrent les résultats d'une intervention sur des aspects cognitifs et conatifs liés à la lecture et à l'écriture.

9 L'adaptation scolaire des enfants intellectuellement précoces. Un effet paradoxal du cognitif sur le conatif? est la question à laquelle $P$. Vrignaud tente de répondre à partir des données disponibles sur les aspects conatifs des enfants intellectuellement précoces.

10 À coté des articles traitant de variables classiques du domaine conatif on trouve des variables plutôt psychosociales "étudiées par elles-mêmes ou médiatisées par des variables conatives ». Dans ce contexte, Y. Tazouti montre dans Étude des relations entre appartenance sociale, éducation familiale et performances scolaire: différentes stratégies de recherche, que les variables explicatives de la performance scolaires ne se comportent pas de la même manière dans différents contextes socio-économiques.

11 D'une autre perspective, plus liée à la relation entre enseignant et élève, P. A. Genoud, montre dans la Pertinence et limites des démarches faisant appel aux perceptions subjectives des élèves, une contribution à l'amélioration de la relation maître-élève, en soulignant les avantages de cet outil ainsi que ses limites et contraintes. À son tour, R.-V. Joule dans De la théorie de l'engagement à la pédagogie de l'engagement donne des pistes pour appliquer les principes théoriques de l'engagement dans le contexte scolaire.

Du domaine de la psychologie de la personnalité, C. Kindelberger, J. van Zwieten et S. Branje apportent une analyse sur la «place de la personnalité dans les relations avec les pairs à l'adolescence ", vu sa contribution à l'ajustement socio-émotionnel et scolaire des élèves (Gifford-Smith \& Brownell, 2003). Dans une perspective proche, C. Kindelberger et $\mathrm{N}$. Le Floc'h abordent des aspects plutôt externes à la démarche 
scolaire qui pourraient influencer, d'une manière directe ou indirecte, les processus cognitifs à l'école, dans leur communication intitulée, Les activités extrascolaires un facteur négligé de l'adaptation scolaire des enfants et des adolescents.

13 A. Ndobo et al., contribuent à partir de la sociologie à l'étude des Effets différenciés des processus d'identification au lieu et aux pairs en contexte scolaire, et enfin, S. Brunot montre le rôle médiateur des variables conatives liées au soi dans l'impact des contextes sociaux sur les performances cognitives scolaires.

À notre avis, ce livre a principalement deux vertus. Outre la contribution spécifique à chacun des champs disciplinaires, cet ensemble d'articles rappelle, d'une part, que l'amélioration des apprentissages à l'école ne résulte pas d'un seul et unique groupe de variables «magiques", mais qu'il faut considérer plusieurs volets. D'autre part, cet ouvrage s'avère comme un outil de vulgarisation des connaissances scientifiques relatives aux des aspects conatifs, ainsi que pour donner des pistes d'intervention pour favoriser la réussite scolaire.

\section{BIBLIOGRAPHIE}

ATKINSON J.W. (1983). Personality, motivation, and action : Selected papers. New-York : Preager.

GifFoRD-SMith M. E. \& BRownell C. A. (2003). « Childhood peer relationship : social acceptance, friendship and peers networks ». Journal of School Psychology, $n^{\circ} 41$, p. 235-284.

\section{AUTEURS}

JORGE VALENZUELA

Université Catholique de Louvain 